Advance Journal of Food Science and Technology 14(6): 181-185, 2018

DOI:10.19026/ajfst.14.5979

ISSN: 2042-4868; e-ISSN: 2042-4876

(C) 2018 Maxwell Scientific Publication Corp.

Submitted: June 5, 2018

Accepted: July 3, 2018

Published: October 25, 2018

\title{
Research Article \\ Antibacterial and Anti-Quorum Sensing Activities of Ethanol Extract and its Fractions from Six Wild Fruits of Burkina Faso
}

\author{
Ablassé Rouamba, Moussa Compaoré, Vincent Ouédraogo and Martin Kiendrebeogo \\ Laboratory of Applied Biochemistry and Chemistry (LABIOCA), UFR-SVT, University Ouaga 1 Pr \\ Joseph KI-ZERBO, 03 BP 7021 Ouagadougou 03, Burkina Faso
}

\begin{abstract}
The objective is to highlight the useful of tropical wild edible fruits with antibacterial properties. Six wild edible fruits from medicinal plant of Burkina Faso were extracted by ethanol and fractioned by using hexane, chloroform, ethyl acetate and methanol. The samples antibacterial effect was evaluated by using agar diffusion method and their anti-quorum sensing activity was measured spectrophotometrically. The bioactive compounds like flavonoid and polyphenolic compounds were quantified in different fractions from fruits. Interestingly, fruit extracts and fractions inhibited the growth of Pseudomonas aeruginosa POA1 tetracycline resistant and Staphylococcus aureus aztreonam resistant but the fractions did not improve the diameter of inhibition zone. According to this data, extracts and salicylic acid at $100 \mu \mathrm{g} / \mathrm{mL}$ compared to vehicle didn't affect bacterial growth but exhibited an antiquorum sensing activity by inhibiting pyocyanin production and elastase activity. The pyocyanin production inhibition was increased from 11.68 to $54.54 \%$ and the elastase activity inhibition, from 21.05 to $40.35 \%$. Interestingly, extract of Adansonia digitata was more active on the inhibition of pyocyanin production than salicylic acid while extract of Vitellaria paradoxa presented the same inhibition of elastase activity like salicylic acid ( $>0.05$ ). The phenolic compounds contribution to the diameter of inhibition zone of Pseudomonas aeruginosa, elastase activity and pyocyanin production inhibition was $0.56,0.55$ and $0.76(\mathrm{p}<0.05)$ respectively. These findings showed that tropical wild edible fruits are a reservoir of antibacterial bioactive compounds and could be exploited to fight microbial resistance.
\end{abstract}

Keywords: Bioactive compounds, edible fruit, elastase activity, microbial resistance, pyocyanin production, quorum sensing

\section{INTRODUCTION}

Antimicrobial resistance is a significant public health threat and a global crisis. According to WHO statistical investigations, significant morbidity and mortality have been associated to infections with antibiotic-resistant organisms (World Health Organization, 2014). In west Africa, the situation is startling due to the existence of a lot of barriers notably the gaps of data in antimicrobial resistance, surveillance networks and the existence of street medication (Percival et al., 2015). The antimicrobial resistance rate of Escherichia coli, Klebsiella pneumoniae and Klebsiella spp. Non-Typhoidal Salmonella (NTS), Salmonella enterica serotype Typhi, Streptococcus pneumoniae and Staphylococcus aureus was around at 90\% according to Bernabe et al. (2017) data.

In front of this invoking and complex situation, the researchers promoted the use of traditional medicinal plants as an alternative solution in the low income countries. In tropical regions, wild edible fruits are not only used in the human diet but are also employed in the folklore system of medicine for the treatment of microbial infections. The fruits pulp of Adansonia digitata L. (Bonbacaceae), Detarium microcarpum Guill. and Perr. (Caesalpiniaceae), Saba senegalensis (A. Dc) Pichon var. (Apocynaceae), Vitellaria paradoxa C.F. Gaertn. (Sapotaceae), Ziziphus mauritiana Lam. (Rhamnaceae) and Parkia biglobosa (Jacq.) R. Br. (Anacardiaceae) are used in tropical regions to treat dermatitis, dysentery, tuberculosis, meningitis, jaundice (Nadembega et al., 2011; Akah et al., 2012; Ajiboyea et al., 2014; Sundarambal et al., 2015). The previous antimicrobial demonstration of these medicinal plants was mainly focalized on the antibacterial and antifungal abilities of extracts from stem bark, bark, seed and leaves extracts (Akah et al., 2012; Igwo-Ezikpe et al., 2013). However, in some

\footnotetext{
Corresponding Author: Moussa Compaoré, Laboratory of Applied Biochemistry and Chemistry (LABIOCA), UFR-SVT, University Ouaga 1 Pr Joseph KI-ZERBO, 03 BP 7021 Ouagadougou 03, Burkina Faso, Tel.: +226 70064742
}

This work is licensed under a Creative Commons Attribution 4.0 International License (URL: http://creativecommons.org/licenses/by/4.0/). 
previous studies, the hydro ethanol and aqueous extracts of fruit pulp of $D$. microcarpum were able to inhibit bacteria development (Kini et al., 2010), ethanol extract from $P$. biglobosa pods inhibited $S$. aureus and $P$. aeruginosa growth (Igwo-Ezikpe et al., 2013), chloroform and methanol fraction from Z. mauritiana fruit pulp regulated $S$. aureus growth (Beg et al., 2016). The present investigation highlights the useful of tropical wild edible fruits with antimicrobial abilities. It was evaluated the Diameter of Inhibition Zone (DIZ) and the inhibition of Pseudomonas aeruginosa virulence factors production that were pyocyanin and elastase. The bioactive compounds like flavonoid and polyphenolic compounds were quantified in different organic fractions and ethanol extract.

\section{MATERIALS AND METHODS}

Chemicals and reagents: Folin-Ciocalteu reagent, Aluminum trichloride, Salicylic acid, Dimethyl Sulfoxide (DMSO), Luria Bertani (LB) agar, Elastin Congo Red (ECR) and Sodium carbonate were purchased from Sigma-Aldrich (Germany). Methanol, Ethanol, Chloroform, Ethyl acetate and Hexane were purchased by Prolabo (Paris, France). Silica gel® 60 $\mathrm{F}_{254}, 60 \AA$, Tetracycline 0 and Aztreonam were purchased by Merck, Germany. All solvents used were analytical grades.

Bacteria origin: Pseudomonas aeruginosa PAO1 and Staphylococcus aureus ATCC 25923 were provided respectively from the Laboratoire de Biotechnologie Végétale (Université Libre de Bruxelles, Gosselies, Belgium) and the Centre National de Recherches Scientifiques et Technologies (Burkina Faso). $P$. aeruginosa PAO1 and S. aureus $\left(37^{\circ} \mathrm{C}\right.$, agitation 175 rpm) were grown in LB broth.

Samples preparation: Fruits of wild plants Detarium microcarpum, Adansonia digitata, Saba senegalensis, Vitellaria paradoxa, Parkia biglobosa and Ziziphus. mauritiana were collected in Gampela region $(25 \mathrm{Km}$, east of Ouagadougou, Burkina Faso). Fruits of $V$. paradoxa, $P$. biglobosa and $S$. senegalensis were collected during a winter period between June and August and fruits of D. microcarpum, Z. mauritiana and $A$. digitata were collected between January and March period. Fruits were also botanically identified in the laboratory of vegetal biology and ecology of the University Ouaga 1 Pr Joseph KI-Zerbo and some voucher herbal were deposited at department herbarium (D. microcapum CI: 15928, A. digitata CI: 15929, Z. mauritiana CI: 15930, P. biglobosa CI: 15931, V. paradoxa CI: 15932, S. senegalensis CI:15933). Within $4 \mathrm{~h}$ after harvesting, the fruits were washed with sterilized water and air dried after which pulps were scrapped and powdered for extraction.

Pulp powder of each fruit was soaked in ethanol (24 h, $25^{\circ} \mathrm{C}$, continuous stirring). Extract was filtrated, concentrated to dryness in a vacuum evaporator. For the fractionation, $1 \mathrm{~g}$ of each fruit ethanol extract was adsorbed in $10 \mathrm{~g}$ of silica gel (Silicagel ${ }^{\circledR} 60 \mathrm{~F}_{254}, 60 \AA$ ) and washed three times successively with hexane, chloroform, ethyl acetate and methanol. The fractions, obtained after filtration were evaporated to dryness and solubilized in adequate solvent for the phytochemical and anti-microbial investigations.

\section{Antimicrobial assay:}

Agar diffusion method: The sensitivity of different bacterial strains $(S$. aureus and $P$. aeruginosa, with optical density $0.02 \mathrm{~nm}$ ) to ethanol crud extract and fractions of each fruit was measured by using disc diffusion method (Chingizova et al., 2017). Sterilized discs impregnated with each extract fraction (100 $\mu \mathrm{g} /$ disc) were incubated in Petri plates containing Luria Bertani agar spread with the inoculum $\left(10^{6}\right.$ cellules/mL). Negative solvent controls (1\% of DMSO) and positive controls antibiotic Tetracycline 0 and Aztreonam (30 $\mu \mathrm{g} /$ disc) were used. After $24 \mathrm{~h}$ of incubation at $37^{\circ} \mathrm{C}$, the Diameters of Zones of Inhibition (DIZ) from sample action were measured.

Inhibition of pyocyanin production and elastase activity: The anti-quorum sensing activity of ethanol crud extract was assessed by measuring the pyocyanin production and the elastase activity in $P$. aeruginosa PAO1 culture. The pyocyanin quantification was assessed by using the method described by Krishnan et al. (2012). Briefly, a single colony of P. aeruginosa PAO1 culture was inoculated in $1 \mathrm{~mL}$ nutrient broth containing each sample $(100 \mu \mathrm{g} / \mathrm{mL})$ and control tubes without sample were also maintained. After $24 \mathrm{~h}$ of incubation at $37^{\circ} \mathrm{C}$, each tube was centrifuged at $3000 \mathrm{~g}$ for $10 \mathrm{~min}$ and pyocyanin was extracted with chloroform in the supernatant. The pyocyanin production was estimated at $520 \mathrm{~nm}$ and the inhibition percentages were calculated.

The inhibition of elastase activity of samples in culture supernatants was evaluated by using Elastin Congo Red method (ECR) as described by Adonizio et al. (2008). Briefly, culture supernatant mixed with ECR was incubated for $16 \mathrm{~h}$ and insoluble ECR was removed by centrifugation (3000 g, $5 \mathrm{~min}$ ). The elastase activity was estimated at $415 \mathrm{~nm}$ and the inhibition percentages of elastase activity were calculated. Salicylic acid was used as reference compound. To assess the effect of samples on bacterial growth, the level of growth of $P$. aeruginosa PAO1 in the pellet was compared to the control (without sample) by measuring the optical density at $600 \mathrm{~nm}$.

Polyphenolic content determination: The total phenolic and flavonoid contents were estimated by using the standard colorimetric method (Compaoré et al., 2016). The standard calibration curve ( $\mathrm{y}=201 \mathrm{x}$ 21,$\left.22 ; \mathrm{R}^{2}>0.99 ; \mathrm{p}<0.0001\right)$ was generated by using 
Gallic acid $(0-100 \mu \mathrm{g} / \mathrm{mL})$ and total phenolic content was expressed as mg gallic acid equivalent to $10 \mathrm{~g}$ of fruit extract (mg GAE/10 g). Quercetin (0-100 mg/L) was used to plot a standard calibration curve $(\mathrm{y}=39.8 \mathrm{x}$ - 3.5; $\mathrm{R}^{2}=0.99 ; \mathrm{p}<0.0001$ ) and total flavonoid content was expressed as $\mathrm{mg}$ of quercetin equivalent to $10 \mathrm{~g}$ of plant extract (mg QE/10 g). The data were obtained in triplicate test for each sample.

Statistical analysis: Experiments were performed in triplicate $(n=3)$ and data were presented as mean \pm S.D. Graph Pad Software (Graph Pad Software Inc., San Diego, CA, USA) was used for statistical analyses. The One-way ANOVA for repeated measures followed by Newman-Keuls post-test was used to verify the statistical difference on phenolic contents between extracts and the impact of extracts on bacterial growth, pyocyanin and elastase production. p value $<0.05$ was considered as being significant.

\section{RESULTS}

Diameters of Zone Inhibition (DIZ): The diameters of inhibition zones of samples were indicated in the
Table 1. P. aeruginosa was more sensitive than $S$. aureus according to this data. Z. mauritiana and $S$. senegalensis extract and fractions would contain the best molecules inhibitor of $P$. aeruginosa and $S$. aureus growth. Probably, these bioactive molecules were extracted by ethanol, methanol, ethyl acetate and hexane. Chloroform fraction was the least active. Remarkably, the fractionation didn't increase the diameter of zone inhibition because all extract presented a better DIZ than fractions $(\mathrm{p}>0.05)$. All fractions and extracts of fruits exhibited a weak antimicrobial activity than references active antibiotics. Interestingly, fruit extracts and fractions could inhibit $P$. aeruginosa tetracycline resistant and $S$. aureus aztreonam resistant. These finding suggested that these extracts could be a potential source of new promoted antimicrobial compounds.

Anti-quorum sensitive activities: The anti-quorum effect of ethanol extracts was presented in the Table 2. According to this data, all extracts, salicylic acid and vehicles were showed the similar bacterial turbidity ( $>0.05)$ suggesting that extracts and salicylic acid (100 $\mu \mathrm{g} / \mathrm{mL}$ ), didn't affect $P$. aeruginosa growth.

Table 1: Inhibition of bacteria growth ability and total polyphenolic content of fractions

\begin{tabular}{|c|c|c|c|c|c|}
\hline \multirow[b]{2}{*}{ Species } & \multirow[b]{2}{*}{ Extract/fractions } & \multicolumn{2}{|c|}{ Diameter of inhibition zones (mm) } & \multicolumn{2}{|c|}{ Total polyphenolic content } \\
\hline & & P. aeruginosa (Gram-) & S. aureus (Gram+) & TFC (mgQE/10 g) & TPC (mgGAE/10g) \\
\hline \multirow[t]{5}{*}{ A. digitata } & Hexane fraction & $10.00 \pm 0.00^{\mathrm{g}}$ & $8.50 \pm 0.71^{j}$ & $3.85 \pm 0.07^{t}$ & $200.38 \pm 11.67^{\mathrm{r}}$ \\
\hline & Chloroform fraction & Non-active & Non-active & $34.54 \pm 1.86^{\mathrm{m}}$ & $2106.96 \pm 8.49^{\mathrm{h}}$ \\
\hline & Ethyl acetate fraction & $9.50 \pm 0.71^{\mathrm{h}}$ & $9.50 \pm 0.71^{\mathrm{g}}$ & $14.68 \pm 0.14^{\circ}$ & $1612.62 \pm 40.89^{j}$ \\
\hline & Methanol fraction & $10.00 \pm 1.41^{\mathrm{g}}$ & Non-active & $50.15 \pm 0.69^{j}$ & $3683.15 \pm 21.11^{\mathrm{d}}$ \\
\hline & Ethanol extract & $11.25 \pm 0.50^{\mathrm{d}}$ & $10.75 \pm 0.35^{\mathrm{e}}$ & $104.20 \pm 2.76^{\mathrm{f}}$ & $7604.10 \pm 20.54^{\mathrm{a}}$ \\
\hline \multirow[t]{5}{*}{ D. microcarpum } & Hexane fraction & $10.50 \pm 0.71^{\mathrm{f}}$ & $8.50 \pm 0.71^{\mathrm{j}}$ & $9.88 \pm 3.68^{\mathrm{q}}$ & $71.69 \pm 5.04^{\mathrm{s}}$ \\
\hline & Chloroform fraction & Non-active & Non-active & $42.63 \pm 3.27^{1}$ & $52.71 \pm 4.07^{\mathrm{t}}$ \\
\hline & Ethyl acetate fraction & Non-active & $8.50 \pm 0.71^{j}$ & $195.22 \pm 3.03^{\mathrm{b}}$ & $1480.28 \pm 15.64^{\mathrm{k}}$ \\
\hline & Methanol fraction & $9.50 \pm 0.71^{\mathrm{h}}$ & $8.00 \pm 0.00^{\mathrm{k}}$ & $10.14 \pm 0.43^{\mathrm{q}}$ & $394.01 \pm 12.59^{\circ}$ \\
\hline & Ethanol extract & $10.83 \pm 0.28^{\mathrm{e}}$ & $13.50 \pm 0.50^{\mathrm{b}}$ & $257.87 \pm 1.68^{\mathrm{a}}$ & $1998.68 \pm 9.25^{\mathrm{i}}$ \\
\hline \multirow[t]{5}{*}{ P. biglobosa } & Hexane fraction & $10.00 \pm 0.00^{g}$ & $9.50 \pm 0.71^{\mathrm{g}}$ & $51.40 \pm 0.90^{\mathrm{j}}$ & $278.32 \pm 23.93^{\mathrm{q}}$ \\
\hline & Chloroform fraction & $9.50 \pm 0.71^{\mathrm{h}}$ & Non-active & $43.83 \pm 3.45^{1}$ & $454.25 \pm 17.79^{\mathrm{n}}$ \\
\hline & Ethyl acetate fraction & Non-active & Non-active & $36.27 \pm 2.13^{\mathrm{m}}$ & $2008.78 \pm 89.66^{\mathrm{i}}$ \\
\hline & Methanol fraction & Non-active & Non-active & $5.51 \pm 0.24^{\mathrm{s}}$ & $373.28 \pm 13.93^{\mathrm{P}}$ \\
\hline & Ethanol extract & $10.00 \pm 0.28^{g}$ & $11.25 \pm 0.50^{\mathrm{d}}$ & $137.01 \pm 1.92^{\mathrm{d}}$ & $3114.63 \pm 35.75^{\mathrm{e}}$ \\
\hline \multirow[t]{5}{*}{ S. senegalensis } & Hexane fraction & $9.50 \pm 0.71^{\mathrm{h}}$ & Non-active & $67.15 \pm 4.35^{\mathrm{h}}$ & $390.03 \pm 29.30^{\text {o.p }}$ \\
\hline & Chloroform fraction & $9.50 \pm 0.71^{\mathrm{h}}$ & $8.50 \pm 0.71^{\mathrm{j}}$ & $25.58 \pm 0.20^{\mathrm{n}}$ & $2329.90 \pm 13.26^{g}$ \\
\hline & Ethyl acetate fraction & $10.50 \pm 0.71^{\mathrm{f}}$ & Non-active & $5.82 \pm 0.13^{\mathrm{s}}$ & $679.21 \pm 39.08^{1}$ \\
\hline & Methanol fraction & $14.50 \pm 0.71^{\mathrm{b}}$ & $9.00 \pm 0.00^{\mathrm{i}}$ & $7.18 \pm 0.10^{r}$ & $378.56 \pm 15.21^{\mathrm{p}}$ \\
\hline & Ethanol extract & $8.16 \pm 0.28^{1}$ & $11.66 \pm 0.28^{c}$ & $105.73 \pm 1.20^{\mathrm{f}}$ & $3777.10 \pm 24.00^{c}$ \\
\hline \multirow[t]{5}{*}{$V$. paradoxa } & Hexane fraction & Non-active & Non-active & $26.64 \pm 1.71^{\mathrm{n}}$ & $73.40 \pm 2.03^{\mathrm{s}}$ \\
\hline & Chloroform fraction & Non-active & Non-active & $63.74 \pm 1.75^{\mathrm{i}}$ & $439.09 \pm 18.20^{\mathrm{n}}$ \\
\hline & Ethyl acetate fraction & $9.00 \pm 0.00^{\mathrm{j}}$ & Non-active & $94.38 \pm 6.87^{g}$ & $1959.19 \pm 84.42^{\mathrm{i}}$ \\
\hline & Methanol fraction & Non-active & $9.50 \pm 0.71^{\mathrm{g}}$ & $7.31 \pm 0.24^{\mathrm{r}}$ & $413.94 \pm 16.68^{\circ}$ \\
\hline & Ethanol extract & $9.25 \pm 0.76^{\mathrm{i}}$ & $10.50 \pm 0.50^{\mathrm{f}}$ & $192.02 \pm 8.58^{\mathrm{c}}$ & $2885.62 \pm 30.25^{f}$ \\
\hline \multirow[t]{5}{*}{ Z. mauritiana } & Hexane fraction & $9.50 \pm 0.71^{\mathrm{h}}$ & $10.50 \pm 0.71^{\mathrm{f}}$ & $46.07 \pm 2.14^{\mathrm{k}}$ & $196.74 \pm 15.43^{\mathrm{r}}$ \\
\hline & Chloroform fraction & $8.50 \pm 0.71^{\mathrm{k}}$ & Non-active & $10.92 \pm 1.06^{\mathrm{q}}$ & $625.58 \pm 18.75^{\mathrm{m}}$ \\
\hline & Ethyl acetate fraction & $9.00 \pm 1.41^{\mathrm{j}}$ & $9.00 \pm 0.00^{\mathrm{i}}$ & $43.99 \pm 0.49^{1}$ & $2816.77 \pm 77.62^{f}$ \\
\hline & Methanol fraction & $10.00 \pm 0.00^{\mathrm{g}}$ & $8.50 \pm 0.71^{\mathrm{j}}$ & $22.35 \pm 1.95^{\mathrm{n}}$ & $1648.55 \pm 39.08^{j}$ \\
\hline & Ethanol extract & $11.83 \pm 0.28^{\mathrm{c}}$ & $9.16 \pm 0.28^{\mathrm{h}}$ & $123.5 \pm 1.41^{\mathrm{e}}$ & $5287.64 \pm 37.71^{\mathrm{b}}$ \\
\hline \multirow{2}{*}{$\begin{array}{l}\text { Standards } \\
\text { antibiotic }\end{array}$} & Tetracycline & Non-active & $26.00 \pm 1.41^{\mathrm{a}}$ & & \\
\hline & Aztreonam & $20.50 \pm 0.71^{\mathrm{a}}$ & Non-active & & \\
\hline
\end{tabular}


Adv. J. Food Sci. Technol., 14(6): 181-185, 2018

Table 2: Anti-quorum sensing activity of fruits extracts

\begin{tabular}{llll}
\hline Sample $(100 \mu \mathrm{g} / \mathrm{mL})$ & Bacterial turbidity $\mathrm{p}>0.05$ & $\begin{array}{l}\text { Inhibition of pyocyanin } \\
\text { production }(\%)\end{array}$ & $\begin{array}{l}\text { Inhibition of elastase activity } \\
(\%)\end{array}$ \\
\hline Vehicle $(\mathrm{DMSO})$ & $0.77 \pm 0.04$ & Non-active & Non-active \\
A. digitata & $0.83 \pm 0.08$ & $54.54 \pm 3.00^{\mathrm{e}}$ & $21.05 \pm 2.00$ \\
D. microcarpum & $0.75 \pm 0.03$ & $25.97 \pm 1.00^{\mathrm{c}}$ & $21.05 \pm 2.00$ \\
P. biglobosa & $0.76 \pm 0.03$ & Non-active & Non-active \\
S. senegalensis & $0.77 \pm 0.04$ & $10.39 \pm 2.00^{\mathrm{d}}$ & $33.33 \pm 2.00^{\mathrm{c}}$ \\
V. paradoxa & $0.74 \pm 0.03$ & $11.68 \pm 1.00^{\mathrm{d}}$ & $45.61 \pm 2.00^{\mathrm{a}}$ \\
Z. mauritiana & $0.77 \pm 0.06$ & $24.67 \pm 1.00^{\mathrm{c}}$ & $40.35 \pm 1.00^{\mathrm{b}}$ \\
Salicylic acid & $0.80 \pm 0.05$ & $35.04 \pm 2.00^{\mathrm{b}}$ & $38.60 \pm 3.00^{\mathrm{b}}$ \\
\hline
\end{tabular}

Values are expressed as mean \pm S.D. ( $\mathrm{n}=3$ independent experiments); Values within each column with different superscripted letters differ significantly $(\mathrm{p}<0.05)$ as determined by ANOVA

The pyocyanin production inhibition was increased from 11.68 to $54.54 \%$ and the elastase activity inhibition was from 21.05 to $40.35 \%$. P. biglobosa extract wasn't showed any anti-quorum activity. Interestingly, extract of $A$. digitata extracts inhibited more pyocyanin production than salicylic acid while extract from $V$. paradoxa presented the same inhibition of elastase activity than salicylic acid $(\mathrm{p}>0.05)$. All these findings suggested that the inhibition of pyocyanin production or elastase activity by the different fruits extracts would not due to the inhibition of bacterial growth but a plausible interference of extract with the quorum sensing system of bacteria thus inhibiting pyocyanin production and elastase activity.

Polyphenolic content evaluation: Fractions and extracts were screened for their total flavonoids and total phenolic contents (Table 1). The flavonoid content was increased form $3.85 \mathrm{mgQE} / 10 \mathrm{~g}$ (A. digitata hexane fraction) to $257.87 \mathrm{mgQE} / 10 \mathrm{~g}$ ( $D$. microcarpum ethanol extract) and the phenolic content was increased from $52.71 \mathrm{mgGAE} / 10 \mathrm{~g}$ (D. microcarpum chloroform fraction) to 7604.10 mgGAE/10 g (Ethanol extract form A. digitata). According to the flavonoid content of ethanol extracts, it was found this order: D. microcarpum $>\mathrm{Z}$. mauritiana $>V$. paradoxa $>A$. digitata (or $S$. senegalensis) $>P$. biglobosa. But according to the phenolic content of ethanol extract it was found the following order: A. digiata $>Z$. mauritiana $>S$. senegalensis $>P$. biglobosa $>V$. paradoxa $>D$. microcarpum. The fractionation demonstrated the notable variability of polarity of metabolites in the ethanol extracts.

\section{DISCUSSION}

This present investigation demonstrated the importance of the use of wild fruits in low income countries for health care. The fruits use could be a promote strategy to fight the bacteria resistance of human pathogenic microbes according to the inhibition of bacterial growth and the anti-quorum sensing effects of ethanol extracts and its fractions of 6 medicinal plant form Burkina Faso. It is the first time that anti-quorum sensing activities of extracts from A. digiata, $Z$. mauritiana, $S$. senegalensis, $P$. biglobosa, V. paradoxa, D. microcarpum were demonstrated.
The growth inhibition of $S$. aureus and $P$. aeruginosa found in the work confirmed the previous antimicrobial investigations. Indeed, aqueous extract from $D$. microcarpum fruit pulp inhibited $S$. aureus mhh 65.8T growth (Kini et al., 2010), ethanol extract of pods from $P$. biglobosa inhibited $S$. aureus and $P$. aeruginosa growth (Igwo-Ezikpe et al., 2013), chloroform and methanol fraction of Z. mauritiana fruit pulp presented a relative inhibition of $S$. aureus growth (Beg et al., 2016). The phytochemical investigation showed high metabolites contents notably flavonoids and phenolic in pulp fruits which was in agreement with previous studies where methanol extract of these 6 fruits pulp showed the highest flavonoids and phenolic contents (Lamien-Meda et al., 2008). In this investigation, flavonoids and phenolic contributed significantly to the anti-quorum sensing activity and to the inhibition of bacteria growth of ethanol extracts and its fractions from 6 fruits. So, the phenolic compound contribution to the DIZ of $P$. aeruginosa, elastase activity and pyocyanin production inhibition was 0.56 ; 0.55 ; and $0.76(\mathrm{p}<0.05)$ respectively. The flavonoid contributed significantly to the DIZ of $P$. aeruginosa only (0.63). The contribution of flavonoids and other phenolic compounds to the anti-quorum sensing activity and to the DIZ of bacteria was previously demonstrated. So, quercetin-3-Glycoside, rutin and quercetin found in tropical wild berries were responsible of the inhibition of $S$. aureus and $P$. aeruginosa growth (Radovanovic et al., 2013). Moreover, phenol acids such as gallic acid and cafeic acid have been reported to inhibit QS-regulated virulence factors expression in $P$. aeruginosa (Rodrigues et al., 2016).

Regarding to the central role of quorum sensing in bacterial virulence, inhibition of bacterial virulence factors production such as pyocyanin and elastase constitute an alternative and effective strategy to attenuate pathogenicity of bacteria resistant to available antibiotics. The effectiveness of fruits pulp extracts and their fractions in inhibition of bacterial growth and virulence factors production, could justify the traditional use of these fruits in West Africa in the treatment of several microbial diseases like dysentery, tuberculosis and meningitis (Sundarambal et al., 2015; Akah et al., 2012). 


\section{CONCLUSION}

Wild edible fruits are rich in antibacterial compounds including total phenolic and total flavonoid. These phytochemicals constituents are both bactericidal potent and quorum sensing inhibitors. The pulp of wild edible fruits constitutes a reservoir of potential antimicrobial compounds for fighting microbial resistance. These funding show that wild edible fruits can be exploited for both their nutraceutical and their therapeutic potential.

\section{ACKNOWLEDGMENT}

The authors gratefully acknowledge the International Foundation for Science (IFS), Stockholm, for supporting the present work under the IFS Grants F/5539-1 and the World Academy of Science (TWAS), Trieste under the grant TWAS 12-044 RG/BIO/AF/AC_G.

\section{REFERENCES}

Adonizio, A., K.F. Kong and K. Mathee, 2008. Inhibition of quorum sensing-controlled virulence factor production in pseudomonas aeruginosa by south florida plant extracts. Antimicrob. Agents Ch., 52(1): 198-203.

Ajiboye, T.O., A.O. Adeleye, A.K. Salau, O.B. Ojewuyi, N.S. Adigun et al., 2014. Phenolic extract of Parkia biglobosa fruit pulp stalls aflatoxin B1 mediated oxidative rout in the liver of male rats. Rev. Bras. Farmacogn., 24(6): 668-676.

Akah, P.A., C.S. Nworu, F.N. Mbaoji, I.A. Nwabunike and C.A. Onyeto, 2012. Genus Detarium: Ethnomedicinal, phytochemical and pharmacological profile. Phytopharmacology, 3(2): 367-375.

Beg, M.A., U.V. Teotia and S. Farooq, 2016. In vitro antibacterial and anticancer activity of Ziziphus. J. Med. Plants Stud., 4(5): 230-233.

Bernabe, K.J., C. Langendorf, N. Ford, J.B. Ronat and R.A. Murphy, 2017. Antimicrobial resistance in West Africa: A systematic review and metaanalysis. Int. J. Antimicrob. Agents, 50(5): 629-639.

Chingizova, E.A., A.V. Skriptsova, M.M. Anisimov and D.L. Aminin, 2017. Antimicrobial activity of marine algal extracts. Int. J. Phytomed., 9: 113122.

Compaoré, M., R.N.T. Meda, S. Bakasso, L. Vlase and M. Kiendrebeogo, 2016. Antioxidative, antiinflammatory potentials and phytochemical profile of Commiphora africana (A. Rich.) Engl. (Burseraceae) and Loeseneriella africana (Willd.) (Celastraceae) stem leaves extracts. Asian Pac. J. Trop. Biomed., 6(8): 665-670.
Igwo-Ezikpe, M.N., H.A. Ogbunugafor, A.P. Gureje and I.J. Ezeonwumelu, 2013. Phytochemical, antioxidant and antimicrobial aroperties of Parkia biglobosa (African locust bean) Pods. The Bioscientist., 1(2): 182-190.

Kini, F., S. Ouédraogo and I.P. Guissou, 2010. Propriétés nutritionnelles et thérapeutiques du fruit de Detarium microcarpum Guill. et Perr. Fruit Vegetable Cereal Sci. Biotechnol., 4(1): 26-30.

Krishnan, T., W.F. Yin and K.G. Chan, 2012. Inhibition of quorum sensing-controlled virulence factor production in Pseudomonas aeruginosa PAO1 by ayurveda spice clove (Syzygium aromaticum) bud extract. Sensors, 12(4): 4016-4030.

Lamien-Meda, A., C.E. Lamien, M.M. Compaoré, R.N. Meda, M. Kiendrebeogo, B. Zeba, J.F. Millogo and O.G. Nacoulma, 2008. Polyphenol content and antioxidant activity of fourteen wild edible fruits from Burkina Faso. Molecules, 13(3): 581-594.

Nadembega, P., J.I. Boussim, J.B. Nikiema, F. Poli and F. Antognoni, 2011. Medicinal plants in Baskoure, Kourittenga province, Burkina Faso: An ethnobotanical study. J. Ethnopharmacol., 133(2): 378-395.

Percival, S.L., L. Suleman, C. Vuotto and G. Donelli, 2015. Healthcare-associated infections, medical devices and biofilms: Risk, tolerance and control. J. Med. Microbiol., 64(Pt 4): 323-334.

Radovanovic, B.C., S.M. Andelkovic, A.B. Radovanovic and M.Z. Andelkovic, 2013. Antioxidant and antimicrobial activity of polyphenol extracts from wild berry fruits grown in Southeast Serbia. Trop. J. Pharm. Res., 12(5): 813819.

Rodrigues, A.C., B.D. de Oliveira, E.R. da Silva, N.T.B. Sacramento, M.C. Bertoldi, U.M. Pinto, 2016. Anti-quorum sensing activity of phenolic extract from Eugenia brasiliensis (Brazilian Cherry). Food Sci. Technol. (Campinas), 36(2): 337-343.

Sundarambal, M., P. Muthusamy, R. Radha and S.A Jerad, 2015. A review on Adansonia digitata Linn. J. Pharmacogn. Phytochem., 4(4): 12-16.

World Health Organization, 2014. Antimicrobial resistance. In Global Report on Surveillance, 1256. ISBN 978924156474 8. Retrieved from: www.who.int/about/licensing/copyright_form/en/in dex.html. 